Tôhoku Math. Journ.

22(1970), 254-269.

\title{
UNIFORMLY ASYMPTOTIC STABILITY \\ AND TOTAL STABILITY
}

\author{
JUNJI KATO*
}

(Received February 2, 1970)

Under the condition that

(c) solutions of every system in the hull are unique for initial conditions, Yoshizawa and the author [1] have shown that a bounded solution of an almost periodic system or, more generally, of a system with the compact hull is totally stable, if it is uniformly asymptotically stable. It is quite natural that there arises a question: Can the condition (c) be omitted or be replaced by a weaker condition? In this article, we shall prove that for autonomous or periodic systems the above assertion is true without the condition (c), and we shall present an example which shows that even for an almost periodic system the assertion is not necessarily true if there is no additional condition such as (c).

1. Let $F(t, \boldsymbol{\phi})$ be an $R^{n}$-valued continuous function defined on $[0, \infty) \times \mathscr{B}$, where $\mathscr{B}$ is a Banach space, and let $T(F)$ be the set of continuous functions $F(t+s, \boldsymbol{\varphi})$ for all $s \geqq 0$, which is a subset of $C\left([0, \infty) \times \mathscr{B}, R^{n}\right)$. Here $C\left(\Omega, R^{n}\right)$ denotes the set of all continuous $R^{n}$-valued functions defined on $\Omega$, and it is a topological space by the compact-open-topology. Let $H(F)$ be the closure of $T(F)$. As will be shown in the below, if $F(t, \phi)$, defined on $(-\infty, \infty) \times \mathscr{B}$, is almost periodic in $t$ uniformly for $\phi \in \mathscr{B}$ and if $\mathscr{B}$ is separable, then $H(F)$ consists of the restriction to $[0, \infty)$ of the elements of the hull in the usual sense. By this reason we may call $H(F)$ the hull of $F$.

The following proposition is well known when $\mathscr{B}$ is a Euclidean space. However it is not trivial for general case, where we should note that the translation numbers can be determined only on each compact subset of $\mathscr{B}$ and that the same argument as in the case where $\mathscr{B}$ is a Euclidean space does not effect, since there may not exist compact subsets $K_{m}$ satisfying $\bigcup_{m} K_{m}=\mathscr{B}$.

Proposition 1. Let $F(t, \phi)$ be almost periodic in $t$ uniformly for $\phi \in \mathscr{B}$, where $\mathcal{B}$ is a separable Banach space. Then, for a given real

(*) This work was partly supported by the U.S.A. National Science Foundation under Grants 7844 and 17601 . 
sequence $\left\{h_{m}\right\}$ there exist a subsequence $\left\{\tau_{m}\right\}$ of $\left\{h_{m}\right\}$ and a function $G(t, \varphi)$ such that the sequence $\left\{F\left(t+\tau_{m}, \varphi\right)\right\}$ converges to $G(t, \varphi)$ uniformly on $(-\infty, \infty) \times S$ for any compact subset $S$ of $\mathscr{B}$, and furthermore, for this $G$ there is a sequence $\left\{\sigma_{m}\right\}, \sigma_{m} \rightarrow \infty$ as $m \rightarrow \infty$, such that $\left\{F\left(t+\sigma_{m}, \boldsymbol{\phi}\right)\right\}$ converges to $G(t, \varphi)$ uniformly on $(-\infty, \infty) \times S$ for any compact subset $S$ of $\mathcal{B}$.

Proof. Since $\mathscr{B}$ is separable, there exists a countable set of points $K_{\infty}=\left\{\boldsymbol{\varphi}^{1}, \boldsymbol{\varphi}^{2}, \cdots\right\}$ which is dense in $\mathcal{B}$. Clearly the $s$ points set $K_{s}=\left\{\boldsymbol{\phi}^{1}, \cdots, \boldsymbol{\phi}^{s}\right\}$ is compact for any $s$. Hence, by the almost periodicity, we can find a subsequence $\left\{\boldsymbol{\tau}_{m}\right\}$ of $\left\{\boldsymbol{h}_{m}\right\}$ such that

(1) $\left\{F\left(t+\tau_{m}, \boldsymbol{\phi}\right)\right\}$ is convergent uniformly on $(-\infty, \infty) \times K_{s}$ for any $s$.

First of all, we shall show that the sequence $\left\{F\left(t+\tau_{m}, \varphi\right)\right\}$ is convergent uniformly on $(-\infty, \infty) \times S$ for any compact subset $S$ of $\mathscr{B}$.

Let $S$ be an arbitrary compact subset of $\mathscr{B}$, and let $\left\{U_{m}^{1}, \cdots, U_{p_{m}}^{m}\right\}$ be a $(1 / m)$-net of $S$. Since the set $K_{\infty}$ is dense in $\mathscr{B}$, there is an integer $k(m, l)$ such that $\phi^{k(m, l)} \in U_{l}^{m}$ for any $m, l$. Let $S^{*}$ be the union of $S$ and the set $\left\{\phi^{k(m, l)} ; l=1, \cdots, p_{m}, m=1,2, \cdots\right\}$. Then we can prove that $S^{*}$ is compact, because $S^{*}$ has no accumulation point outside of the conpact set $S$. As is well known, $F(t, \phi)$ is uniformly continuous on $(-\infty, \infty) \times S^{*}$, and therefore for any $\varepsilon>0$ there exists a number $\delta(\varepsilon)>0$ such that if $\|\varphi-\psi\|<\delta(\varepsilon)$, then

$$
|F(t, \phi)-F(t, \psi)|<\varepsilon
$$

for all $(t, \phi),(t, \psi) \in(-\infty, \infty) \times S^{*}$. Put

$$
N(\varepsilon)=\max \left\{k(m(\varepsilon), l) ; l=1, \cdots, p_{m(\varepsilon)}\right\},
$$

where $m(\varepsilon)$ is an integer greater than $1 / \delta(\varepsilon / 3)$. By $(1)$, there is an integer $N_{\mathrm{o}}(\varepsilon)$ such that if $\mu, \nu \geqq N_{0}(\varepsilon)$,

$$
\left|F\left(t+\tau_{\mu}, \varphi\right)-F\left(t+\tau_{\nu}, \phi\right)\right|<\frac{\varepsilon}{3} \quad \text { for all }(t, \phi) \in(-\infty, \infty) \times K_{N(\varepsilon)} .
$$

For a given $(t, \phi) \in(-\infty, \infty) \times S$ we can find a $\phi^{k} \in S^{*} \bigcap K_{N^{(}(\varepsilon)}$ such that $\left\|\boldsymbol{\phi}-\boldsymbol{\phi}^{k}\right\|<\delta(\varepsilon / 3)$. Hence we have

$$
\left|F\left(t+\tau_{\mu}, \boldsymbol{\phi}\right)-F\left(t+\tau_{\mu}, \phi^{k}\right)\right|<\frac{\varepsilon}{3} \text { for all } \mu .
$$

Therefore we can see that if $\mu, \nu \geqq N_{0}(\varepsilon)$, then 


$$
\begin{aligned}
& F\left(t+\tau_{\mu}, \boldsymbol{\varphi}\right)-F\left(t+\tau_{\nu}, \boldsymbol{\varphi}\right) \\
& \leqq \\
& \leqq
\end{aligned}
$$

This shows that the sequence $\left\{F\left(t+\tau_{m}, \varphi\right)\right\}$ converges to a unique limiting function $G(t, \varphi)$ uniformly on $(-\infty, \infty) \times S$ for any compact subset $S$ of $\mathscr{B}$. Clearly $G(t, \boldsymbol{\varphi})$ is continuous on $(-\infty, \infty) \times \mathscr{B}$ and belongs to the hull in the usual sense. and

By the almost periodicity there is a number $t_{m}^{s}$ with the properties; $t_{m}^{*} \geqq m$

$$
F\left(t+t_{m}^{s}, \phi\right)-F\left(t+\tau_{m}, \varphi\right) \mid \frac{1}{m} \text { for all }(t, \phi) \in(-\infty, \infty) \times K_{s}
$$

where $t_{m}^{*}-\tau_{m}$ is a $(1 / m)$-translation number on $K_{s}$. Setting $\sigma_{m}=t_{m}^{m}$, we shall show that $\left\{F\left(t+\sigma_{m}, \varphi\right)\right\}$ converges uniformly on $(-\infty, \infty) \times K_{\text {s }}$ for any $s \geqq 0$. Then, by the same argument as for $\left\{F\left(t+\tau_{m}, \phi\right)\right\}$, it would be proved that $\left\{F\left(t+\sigma_{m}, \boldsymbol{\varphi}\right)\right\}$ is convergent uniformly on $(-\infty, \infty) \times S$ for any compact subset $S$ of $\mathcal{B}$. We should note that

$$
\left|F\left(t+\sigma_{m}, \boldsymbol{\phi}\right)-F\left(t+\tau_{m}, \boldsymbol{\phi}\right)\right|=\left|F\left(t+t_{m}^{m}, \boldsymbol{\varphi}\right)-F\left(t+\tau_{m}, \boldsymbol{\phi}\right)\right| \leqq \frac{1}{m}
$$

on $(-\infty, \infty) \times K_{s}$ for any $s \leqq m$, since $K_{s} \subset K_{m}$. On the other hand, it follows from (1) that there is a number $N_{s}(\varepsilon)$ such that if $\mu, \nu \geqq N_{s}(\varepsilon)$, then

$$
\left|F\left(t+\tau_{\mu}, \varphi\right)-F\left(t+\tau_{v}, \varphi\right)\right|<\varepsilon \quad \text { for all }(t, \phi) \in(-\infty, \infty) \times K_{s} .
$$

Hence, if $\mu, \nu \geqq \max \left\{N_{s}(\varepsilon / 3), 3 / \varepsilon, s\right\}$, then we have

$$
\begin{aligned}
& F\left(t+\sigma_{\mu}, \varphi\right)-F\left(t+\sigma_{\nu}, \phi\right) \\
& \leqq F\left(t+\sigma_{\mu}, \boldsymbol{\varphi}\right)-F\left(t+\tau_{\mu}, \boldsymbol{\varphi}\right)+F\left(t+\tau_{\mu}, \boldsymbol{\varphi}\right)-F\left(t+\tau_{\nu}, \boldsymbol{\varphi}\right) \\
&+F\left(t+\tau_{\nu}, \boldsymbol{\varphi}\right)-F\left(t+\sigma_{\nu}, \boldsymbol{\varphi}\right)<\frac{1}{\mu}+\frac{\varepsilon}{3}+\frac{1}{\nu} \leqq \varepsilon \quad(\text { by }(2))
\end{aligned}
$$

for all $(t, \varphi) \in(-\infty, \infty) \times K_{s}$, that is, $\left\{F\left(t+\sigma_{m}, \varphi\right)\right\}$ is convergent uniformly on $(-\infty, \infty) \times K_{s}$ for any $s$.

Finally, it follows from (2) that $\left\{F\left(t+\sigma_{m}, \varphi\right)\right\}$ converges to the limiting function $G(t, \varphi)$ of the sequence $\left\{F\left(t+\tau_{m}, \phi\right)\right\}$ on $(-\infty, \infty) \times K_{k}$ for any $s$. Hence $\left\{F\left(t+\sigma_{m}, \boldsymbol{\phi}\right)\right\}$ converges to $G(t, \phi)$ on $(-\infty, \infty) \times \mathscr{B}$, because $K_{\infty}=\bigcup K_{s}$ 
is dense in $\mathscr{B}$ and $G(t, \Phi)$ is continuous on $(\cdots, \infty) \times \mathscr{B}$.

This proposition also shows that if $F(t, \varphi)$ is almost periodic in $t$ uniformly for $\varphi \in \mathscr{B}$, then the hull is a compact subset of $C\left((-\infty, \infty) \times \mathscr{B}, R^{n}\right)$. Actually it is compact under the stronger topology, that is, in the sense of uniform convergence on $(-\infty, \infty) \times S$ for any compact subset $S$ of $\mathscr{B}$. Under the compact-opentopology, we can prove the following.

Proposition 2. Let $F(t, \varphi)$ be a continuous $R^{n}$-valued function on $[0, \infty) \times \mathscr{B}$, where $\mathscr{B}$ is a separable Banach space. Then the hull $H(F)$ is compact if and only if $F(t, \boldsymbol{\phi})$ is uniformly continuous in $(t, \boldsymbol{\phi})$ and bounded on $[0, \infty) \times S$ for any compact subset $S$ of $\mathscr{B}$.

Proor. The proof for the necessity is not difficult. Now we shall show the sufficiency.

Let $\left\{h_{m}\right\}, h_{m} \geqq 0$, be a given sequence. Then, by the assumption, the sequence $\left\{F\left(t+h_{m}, \varphi\right)\right\}$ is a normal family on $[0, \infty) \times K_{x}$ for any $s$, where $K_{s}$ is the set given in the proof of Proposition 1. Hence there is a subsequence $\left\{\boldsymbol{\tau}_{m}\right\}$ of $\left\{\boldsymbol{h}_{m}\right\}$ such that the sequence $\left\{\boldsymbol{F}^{\prime}\left(\boldsymbol{t}+\boldsymbol{\tau}_{m}, \boldsymbol{\phi}\right)\right\}$ is convergent uniformly on $[a, b] \times K_{s}$ for any $s$ and for any compact interval $[a, b] \subset[0, \infty)$. This assertion corresponds to (1). Thus, by using the same arguments as in the proof of Proposition 1, we can prove that $\left\{F\left(t+\tau_{m}, \varphi\right)\right\}$ is convergent uniformly on $[a, b] \times S$ for any compact set $S \subset \mathscr{B}$ and for any compact interval $[a, b] \subset[0, \infty)$. Hence the hull $H(F)$ is compact, since $H(F)=\overline{T(F)}$.

2. Throughout this paper, suppose that $F(t, \varphi)$ is tounded on $[0, \infty) \times \bar{C}_{B^{*}}$. and uniformly continuous in $(t, \phi)$ on $[0, \infty) \times S$ for any compact set $S \subset \bar{C}_{B^{*}}$, where $\bar{C}_{B^{*}}$ denotes the set of $\phi \in C\left([-h, 0], R^{n}\right)$, where $h \geqq 0$ is a fixed constant, with the property

$$
\|\boldsymbol{\phi}\|=\sup \{\boldsymbol{\phi}(\theta) ; \theta \theta[-h, 0]\} \leqq B^{*} .
$$

Since $\left.C(\mid-h, 0], R^{n}\right)$ is a separable Banach space, the hull $H(F)$ is a compact subset of $C\left([0, \infty) \times \bar{C}_{B^{*}}, R^{n}\right)$ by Proposition 2 .

Consider the system of functional differential equations

$$
\dot{x}(t)=F\left(t, x_{l}\right) \text {, }
$$

where $\dot{x}(t)$ denotes the right hand derivative of a given continuous function $x(t)$ and $x_{t}$ denotes an element of $C\left([-h, 0], R^{n}\right)$ defined by

$$
x_{\iota}(\theta)=x(t+\theta) \text { for } \theta \in[-h, 0] .
$$


Then the condition (c) in the introduction means that for every element $G(t, \phi) \in H(F)$ the solutions of

$$
\dot{x}(t)=G\left(t, x_{l}\right)
$$

are unique for initial conditions.

In the sequel, let $u(t)$ be a solution of the system (3) such that

$$
\left\|u_{t}\right\| \leqq B \text { for all } t \geqq 0
$$

and for some $B<B^{*}$. Since $|F(t, \phi)| \leqq L$ for all $(t, \varphi) \in[0, \infty) \times{\overline{C_{B}}}^{*}$ and for some constant $L>0, u(t)$ satisfies the Lipschitz condition

$$
|u(t)-u(s)| \leqq L|t-s| \text { for all } t, s \geqq 0 .
$$

Hence the hulls $H(u)$ and $H(u, F)$ are compact by Proposition 2. Let $(v(t), G(t, \varphi))$ be an element of $H(u, F)$. Then clearly $v(t)$ is a solution of (4). Simply we shall call $v(t)$ a solution in $H(u)$. For example, we shall say that the solutions in $H(u)$ are unique for initial conditions, if for any $(v(t), G(t, \varphi))$ $\in H(u, F) v(t)$ is a unique solution of the system (4) for initial conditions.

The theorem proved in [1] is the following.

THEOREM A. Under the condition (c), uniformly asymptotic stability of $u(t)$ implies its total stability.

Now we shall consider the condition

$\left(\mathrm{c}^{*}\right)$ the solutions in $H(u)$ are uniformly asymptotically stable with a common triple $\left(\delta(\cdot), \delta_{0}, T(\cdot)\right)$,

that is, for any $\varepsilon>0$, any $t_{0} \geqq 0$ and any $(v, G) \in H(u, F)$,

$$
\left\|v_{t_{0}}-x_{t_{0}}\right\|<\delta(\varepsilon) \text { implies }\left\|v_{t}-x_{t}\right\|<\varepsilon \text { for all } t \geqq t_{0}
$$

and

$$
\left\|v_{t_{0}}-x_{t_{0}}\right\| \leqq \delta_{9} \text { implies }\left\|v_{t}-x_{t}\right\|<\varepsilon \text { for all } t \geqq t_{0}+T(\varepsilon),
$$

whenever $x(t)$ is a solution of (4).

Then we have the following theorem. stable.

THEOREM 1. If the condition ( $\left.\mathrm{c}^{*}\right)$ holds good, then $u(t)$ is totally 
We know that if $u(t)$ is uniformly asymptotically stable, then the condition $\left(c^{*}\right)$ holds good for almost periodic systems or, more generally, for systems with compact hulls under the condition (c) and it does for periodic systems without the condition (c) (see [2]). Therefore Theorem A is a corollary of this theorem and we have also the following corollary, which can also be proved by a similar way to that in the proof of Theorem 7.1 in [3].

COROLlARY. A bounded solution of a periodic system is totally stable, if it is uniformly asymptotically stable.

To prove Theorem 1, first of all, we shall give three lemmas. In these lemmas, $g(t)$ is a continuous function defined on $[0, \infty)$ and $y(t)$ is a solution of the system

$$
\dot{y}(t)=F\left(t, y_{t}\right)+g(t) .
$$

LEMMA 1. Suppose that the solutions in $H(u)$ are unique for initial conditions. Let $\varepsilon$ and $T$ be positive constants. Then there exist positive numbers $\delta_{1}(\varepsilon, T)$ and $\delta_{2}(\varepsilon, T)$ such that for any $t_{0} \geqq 0$, if

$$
\left\|y_{t_{0}}-u_{t_{0}}\right\|<\delta_{1}(\varepsilon, T)
$$

and if

$$
|g(t)|<\delta_{2}(\varepsilon, T) \text { on }\left\lfloor t_{0}, t_{0}+T\right\rfloor
$$

then we have

$$
\left\|y_{\iota}-u_{l}\right\|<\varepsilon \text { on }\left[t_{0}, t_{0}+T\right] \text {. }
$$

This lemma can be proved by the same idea as in the proof of Lemma 6 in [2].

LEMMA 2. Let the solutions in $H(u)$ be uniformly stable with a common number $\delta(\cdot)$. Then for any $\varepsilon>0$ and any $T>0$ there are positive numbers $\eta_{1}(\varepsilon)$, independent of $T$, and $\eta_{2}(\varepsilon, T)$ such that for any $t_{0} \geqq 0$, if

$$
\left\|y_{t_{0}}-u_{t_{0}}\right\|<\eta_{1}(\varepsilon)
$$

and if

$$
|g(t)|<\eta_{\imath}\left(\varepsilon, T^{\prime}\right) \text { on }\left[t_{0}, t_{0}+T\right]
$$

then w'e have

$$
\left\|y_{t}-u_{t}\right\|<\varepsilon \text { on }\left[t_{0}, t_{0}+T\right]
$$


ProOF. We can assume that $\varepsilon<B^{*}-B$. Let $K$ be a compact subset of $\bar{C}_{B} *$ such that $u_{t} \in K$ for all $t \geqq 0$ and that

$$
K \supset\left\{\boldsymbol{\varphi} ;\|\boldsymbol{\varphi}\| \leqq B^{*},\left|\boldsymbol{\varphi}(\theta)-\boldsymbol{\varphi}\left(\theta^{\prime}\right) \leqq(L+1)\right| \theta-\theta^{\prime} ; \text { on }[-h, 0]\right\},
$$

where $L$ is a bound for $F(t, \phi)$ on $[0, \infty) \times \bar{C}_{B^{*}}$.

First of all, we shall show that the conclusion of this lemma is true if $y_{t_{0}} \in K$, that is, there exists a number $\eta_{2}^{\prime}(\varepsilon, T)>0$ such that for any $t_{0} \geqq 0$, if $\left\|y_{t_{0}}-u_{\iota_{0}}\right\|<\delta(\varepsilon / 2) / 2, \quad y_{t_{0}} \in K$ and if $|g(t)|<\eta_{2}^{\prime}(\varepsilon, T)$ on $\left[t_{0}, t_{0}+T\right]$, then $\left\|y_{t}-u_{t}\right\|<\varepsilon$ on $\left[t_{0}, t_{0}+T\right]$. Suppose that there is no $\eta_{2}^{\prime}(\varepsilon, T)$. 'Then there exist sequences $\left\{t_{m}\right\}, t_{m} \geqq 0,\left\{g_{m}(t)\right\},\left\{y^{m}(t)\right\},\left\{\tau_{m}\right\}, t_{m} \leqq \tau_{m} \leqq t_{m}+T$, such that

$$
\begin{aligned}
& g_{m}(t)<\frac{1}{m}, \quad y_{t_{m}}^{m}-u_{t_{m}}<\frac{1}{2} \delta\left(\frac{\varepsilon}{2}\right), \quad y_{t_{m}}^{m} \in K, \\
& y_{\tau_{m}}^{m}-u_{\tau_{m}}=\varepsilon, \quad y^{m}{ }_{t}-u_{t}<\varepsilon \text { on }\left[t_{m}, \tau_{m}\right),
\end{aligned}
$$

where $y^{m}(t)$ is a solution, defined on $\left[t_{m}, \tau_{m}\right]$, of the system

$$
\dot{y}(t)=F\left(t, y_{t}\right)+g_{m}(t) .
$$

Clearly we can assume that the sequences

$$
\left\{\left(\boldsymbol{u}\left(t+t_{m}\right), F\left(t+t_{m}, \varphi\right)\right)\right\},\left\{\boldsymbol{\tau}_{m}-t_{m}\right\},\left\{y^{m}\left(t+t_{m}\right)\right\}
$$

are convergent to $(v(t), G(t, \varphi)) \in H(u, F), \sigma \in[0, T]$ and a continuous function $y(t)$, respectively, where we should note that $y^{m}{ }_{t} \in K$ for all $t \in\left[t_{m}, \tau_{m}\right]$. From the assumptions, it turns out that $y(t)$ is also a solution of the system (4) defined on $[0, \sigma]$, and clearly

$$
\left\|y_{0}-v_{0}\right\| \leqq \frac{1}{2} \delta\left(\frac{\varepsilon}{2}\right)<\delta\left(\frac{\varepsilon}{2}\right) \text { and } \| y_{\sigma}-v_{\sigma}=\varepsilon .
$$

This contradicts the uniform stability of $v(t)$. Thus there exists an $\eta_{2}^{\prime}\left(\varepsilon, T^{\prime}\right)$ mentioned in the above.

On the other hand, whatever $y_{t_{0}}$ is, it follows from Lemma 1 that

$$
y_{t_{1}+h} \in K \text { and }\left\|y_{t_{0}+h}-u_{t_{0}+h}\right\|<\frac{1}{2} \delta\left(\begin{array}{c}
\varepsilon \\
2
\end{array}\right)
$$

for any $t_{0} \geq 0$, if $\left\|y_{t_{0}}-u_{t_{0}}\right\|<\delta_{1}(\delta(\varepsilon / 2) / 2, h)$ and $|g(t)|<\min \left\{\delta_{3}(\delta(\varepsilon / 2) / 2, h), 1\right\}$, 
where $\delta_{1}$ and $\delta_{2}$ are those in Lemma 1 .

Therefore, if we set

$$
\eta_{1}(\varepsilon)=\delta_{1}\left(\frac{1}{2} \delta\left(\frac{\varepsilon}{2}\right), h\right), \quad \eta_{2}(\varepsilon, T)=\min \left\{\delta_{2}\left(\frac{1}{2} \delta\left(\frac{\varepsilon}{2}\right), h\right), \eta_{2}^{\prime}(\varepsilon, T), 1\right\}
$$

$\eta_{1}(\varepsilon)$ and $\eta_{2}(\varepsilon, T)$ satisfy the conditions in Lemma 2. This completes the proof.

LEMMA 3. Suppose that the solutions in $H(u)$ are uniformly asymptotically stable with a common triple $\left(\delta(\cdot), \delta_{0}, T(\cdot)\right)$. Then there is a pair $\left(\eta_{3}, \eta_{4}(\cdot)\right)$ with the property: For any $\varepsilon>0$ and any $t_{0} \geqq 0$, if

$$
\left\|y_{t_{0}}-u_{t_{0}}\right\|<\eta_{3}
$$

and if

$$
|g(t)|<\eta_{4}(\varepsilon) \text { on }\left[t_{0}, t_{0}+\tau(\varepsilon)\right]
$$

where $\tau(\varepsilon)=T(\varepsilon / 2)+h$, then the solution $y(t)$ of (5) is continuable on $\left[t_{0}, t_{0}+\tau(\varepsilon)\right]$ and satisfies

$$
\left\|y_{t_{0}+\tau(s)}-u_{t_{0}+\tau(s)}\right\|<\varepsilon
$$

Proof. Suppose that $y_{t_{0}}$ and $g(t)$ satisfy the conditions

$$
\left|y_{t_{0}}-u_{t_{v}} \|<\eta_{1}\left(B^{*}-B\right),\right| g(t) \mid<\eta_{2}\left(B^{*}-B, \tau(\varepsilon)\right),
$$

where $\eta_{1}$ and $\eta_{2}$ are those given in Lemma 2. Then, by Lemma 2,y(t) is continuable to $t_{0}+\tau(\varepsilon)$. The other parts of the proof will be completed by the same arguments as in the proof of Lemma 2. Namely, by putting

$$
\eta_{3}^{\prime}=\min \left\{\delta_{0}, \eta_{1}\left(B^{*}-B\right)\right\}
$$

we can find a positive number $\eta_{4}^{\prime}(\varepsilon) \leqq \eta_{2}\left(B^{*}-B, \tau(\varepsilon)\right)$ such that for any $t_{0} \geqq 0$, $\left\|y_{t_{0}}-u_{t_{0}}\right\|<\eta_{3}^{\prime}$ and $|g(t)|<\eta_{4}^{\prime}(\varepsilon)$ on $\left[t_{0}, t_{0}+T(\varepsilon / 2)\right]$ imply $\left\|y_{t_{0}+T(\varepsilon / 2)}-u_{t_{0}+T(s / 2)}\right\|$ $<\varepsilon$ under the restriction $y_{t_{0}} \in K$, where $K$ is the compact set given in the proof of Lemma 2. Thus it turns out that it is sufficient to set

$$
\eta_{3}=\delta_{1}\left(\eta_{3}^{\prime}, h\right), \eta_{4}(\varepsilon)=\min \left\{\delta_{2}\left(\eta_{3}^{\prime}, h\right), \eta_{2}\left(B^{*}-B, \tau(\varepsilon)\right), \eta_{4}^{\prime}(\varepsilon), 1\right\},
$$


where $\delta_{1}$ and $\delta_{2}$ are those in Lemma 1 .

Proof of Theorem 1. Let $\eta_{1}, \eta_{2}$ be the numbers given in Lemma 2, and let $\eta_{3}, \eta_{4}, \tau$ be those in Lemma 3 . Setting

$$
\rho(\varepsilon)=\min \left\{\eta_{1}(\varepsilon), \eta_{3}\right\}, \eta(\varepsilon)=\min \left\{\eta_{\mathcal{2}}(\varepsilon, \tau(\rho(\varepsilon))), \eta_{4}(\rho(\varepsilon))\right\},
$$

we shall prove that for any $t_{0} \geqq 0$, any solution $y(t)$ of the system (5) satisfies

$$
\left\|y_{t}-u_{t}\right\|<\varepsilon \text { for all } t \geqq t_{0},
$$

if $\left\|y_{t_{0}}-u_{t_{0}}\right\|<\rho(\varepsilon)$ and $|g(t)| \gamma(\varepsilon)$ on $\left[t_{0}, \infty\right)$. From Lemma 2 it follows that.

$$
\left\|y_{t}-u_{t}\right\|<\varepsilon \text { on }\left[t_{0}, t_{0}+\tau(\rho(\varepsilon)) \mid,\right.
$$

because $\left\|y_{t_{0}}-u_{t_{0}}\right\|<\eta_{1}(\varepsilon)$ and $|g(t)|<\eta_{2}(\varepsilon, \tau(\rho(\varepsilon)))$. Furthermore, since $\left\|y_{t_{0}}-u_{t_{v}}\right\|$ $<\eta_{3}$ and $|g(t)|<\eta_{4}(\rho(\varepsilon))$, we have

$$
\left\|y_{t_{0}+\tau(\rho(s))}-u_{t_{0}+\tau(\rho(s)}\right\|<\rho(\varepsilon)
$$

by Lemma 3 . By replacing $t_{0}$ by $t_{0}+\boldsymbol{\tau}(\rho(\varepsilon))$ and by using the same arguments, we can see that

$$
\left\|y_{t}-u_{t}\right\|<\varepsilon \text { on }\left[t_{0}+\tau(\rho(\varepsilon)), t_{0}+2 \tau(\rho(\varepsilon))\right]
$$

and

$$
\left\|y_{l_{\bullet}+2=(\rho(\varepsilon))}-u_{t_{n}+2 \tau(\rho(\varepsilon))}\right\|<\rho(\varepsilon) .
$$

Thus, by repeating the same arguments, we have

$$
\left\|y_{\imath}-u_{t}\right\|<\varepsilon \text { for all } t \geqq t_{0},
$$

that is, the solution $u(t)$ is totally stable.

ln Section 4 we shall give an example which shows that the conjecture

(6) the solutions in $H(u)$ are uniformly asymptotically stable with a common triple $\left(\delta(\cdot), \delta_{0}, T(\cdot)\right)$, if the solution $u(t)$ of $(3)$ is uniformly asymptotically stable

is not true even for almost periodic systems. Furthermore, this example shows 
that we cannot omit the condition (c) in Theorem A without any other supplementary condition. This fact is an immediate consequence of the corollary of Theorem 3 in Section 3.

3. We shall give the definition of stability under disturbances from $H(F)$ with respect to a given compact set $K \subset \bar{C}_{B}$. Define $\rho(G, P)$ for $G \in H(F)$ and $P \in H(F)$ by

$$
\rho(G, P)=\sum_{m=1}^{\infty} \begin{array}{cc}
1 & \rho_{m}(G, P) \\
2^{m} & 1+\rho_{m}(\bar{G}, P)
\end{array},
$$

where

$$
\rho_{m}(G, P)=\sup \{\mid G(t, \phi)-P(t, \phi) ;(t, \phi) \in[0, m] \times K\} .
$$

Here we should note that for the space $C\left([0, \infty) \times K, R^{n}\right) \rho$ introduces a topology equivalent to the compact-open-topology. For convenience, set

$$
\rho^{*}(G, P ; t)=\rho\left(G^{t}, P^{\prime}\right),
$$

where $G^{s}(t, \phi)=G(t+s, \phi) \in T(G)$.

DEFINITION. The solution $u(t)$ of $(3)$ is said to be stable under disturbances from $H(F)$ with respect to $K$, if for any $\varepsilon>0$ there exist positive numbers $\gamma(\varepsilon)$ and $r(\varepsilon)$ such that, for any $s \geqq 0$ and any $T>0$, the solution $x(t)$ of $(4)$ satisfies the relation

$$
\left\|u_{t+s}-x_{t}\right\|<\varepsilon \text { for all } t \in[0, T]
$$

whenever $\left\|u_{s}-x_{0}\right\|<\gamma(\varepsilon), x_{0} \in K, G \in H(F)$ and

$$
\rho^{*}\left(G, F^{s} ; t\right)<\eta(\varepsilon) \text { for all } t \in|0, T| \text {. }
$$

This definition is a natural generalization of the one given in [2] or [4], because we have the following lemma.

Lemma 4. Let $F(t, \phi)$ be almost periodic in $t$ uniformly for $\varphi \in K$. Then there is a positive continuous function $a(\sigma)$ of $\sigma>0, a(0)=0$, such that

$$
\sigma(G, P) \geqq \rho^{*}(G, P ; t) \geqq a(\sigma(G, P))
$$

for all $t \geqq 0$ and any $G, P \in H(F)$, where 


$$
\sigma(G, P)=\sup \{|G(t, \phi)-P(t, \phi)| ;(t, \varphi) \in(-\infty, \infty) \times K\} .
$$

ProOF. The first part of the inequality (8) is clear. We shall prove the second part. Since

$$
\sigma\left(G^{t}, P^{t}\right)=\sigma(G, P)
$$

for all $t$ and all $G, P \in H(F)$, it is sufficient to show the existence of a function $a(\cdot)$ satisfying

$$
\rho(G, P) \geqq a(\sigma(G, P)) .
$$

We know that there is an $l(\varepsilon)>0$ such that any interval of length $l(\varepsilon)$ contains an $\varepsilon$-translation number of $F$, and we can easily see that an $\varepsilon$-translation number of $F$ is so for any $G$ in $H(F)$. From this, it follows that fo any $(t, \phi)$ $\in(-\infty, \infty) \times K$ we can find an $s \in[0, l(\varepsilon)]$ such that

$$
|G(t, \varphi)-G(s, \phi)|<\varepsilon,
$$

whatever $G \in H(F)$ is. Put

$$
a(\sigma)=\begin{array}{cc}
1 & \varepsilon \\
2^{m(s)} & 1+\varepsilon
\end{array} \quad\left(\varepsilon=\frac{\sigma}{3}\right)
$$

where $m(\varepsilon)$ is an integer greater than $l(\varepsilon)$. Then, if $\sigma(G, P)=3 \varepsilon>0$, we can show that

$$
\rho_{m(\varepsilon)}(G, P) \geqq \sigma(G, P)-2 \varepsilon=\varepsilon .
$$

Hence we heve

$$
\rho(G, P) \geqq \frac{1}{2^{m(\varepsilon)}} \frac{\rho_{m(\varepsilon)}(G, P)}{1+\rho_{m(\varepsilon)}(G, P)} \geqq \frac{1}{2^{m(\varepsilon)}} \frac{\varepsilon}{1+\varepsilon}=a(3 \varepsilon)=a(\sigma(G, P)) .
$$

By a slight modification we can make $a(\cdot)$ be continuous. This proves the second part of the inequality (8).

Now we shall prove the following theorems.

THEOREM 2. Let the solution $u(t)$ of the system (3) is totally stable, that is, there exists a $\gamma(\varepsilon)>0$ and an $\eta(\varepsilon)>0$ such that if, for any $t_{0} \geqq 0$,

$$
\left\|u_{t_{0}}-\phi^{n}\right\|<\gamma(\varepsilon) \text { and }|g(t)|<\eta(\varepsilon),
$$


then a solution $y(t)$ of the system (5) through $\left(t_{0}, \phi^{0}\right)$ satisfies

$$
\left\|u_{t}-y_{t}\right\|<\varepsilon \text { for all } t \geqq t_{0} .
$$

Let $K$ be a compact subset of $\bar{C}_{B}$, which is positively invariant for any system in the hull.

Then $u(t)$ is stable under disturbances from $H(F)$ with respect to $K$.

Proof. For an $\varepsilon>0$, suppose that there are a $G \in H(F)$, an $s \geqq 0$, a $T>0$ and a solution $x(t)$ of (4) such that

$$
\begin{gathered}
\left\|u_{s}-x_{n}\right\|<\gamma(\varepsilon), x_{n} \in K, \quad\left\|u_{T+s}-x_{T}\right\|=\varepsilon, \\
\left\|u_{t+s}-x_{t}\right\|<\varepsilon \text { on }[0, T)
\end{gathered}
$$

and

$$
\rho^{*}\left(G, F^{s} ; t\right)<\frac{1}{2} \frac{\eta(\varepsilon)}{1+\eta(\varepsilon)} \text { for all } t \in[0, T]
$$

Since

$$
\rho^{*}\left(G, F^{s} ; t\right)=\rho\left(G^{t}, F^{t+s}\right) \geqq \frac{1}{2} \frac{\rho_{1}\left(G^{t}, F^{t+s}\right)}{1+\rho_{1}\left(G^{t}, F^{t+s}\right)},
$$

it follows from (9) that

$$
\eta(\varepsilon)>\rho_{1}\left(G^{t}, F^{t+s}\right)=\sup \{|G(\tau, \phi)-F(\tau+s, \phi)| ;(\tau, \phi) \in\{t, t+1] \times K\}
$$

for all $t \in[0, T]$. On the other hand, the positive invariance of $K$ implies that $x_{t} \in K$ as long as $x(t)$ exists. Hence we have

$$
\left|G\left(t, x_{t}\right)-F\left(t+s, x_{t}\right)\right|<\eta(\varepsilon) \text { on }[0, T]
$$

or

$$
\left|G\left(t-s, x_{t-s}\right)-F\left(t, x_{t-s}\right)\right|<\eta(\varepsilon) \text { on }[s, s+T] .
$$

Therefore we can easily find a continuous function $g(t)$ which is defined on $[0, \infty)$ and satisfies

$$
g(t)=G\left(t-s, x_{t-s}\right)-F\left(t, x_{t-s}\right) \text { for } t \in[s, s+T \mid
$$

and

$$
|g(t)|<\eta(\varepsilon) \text { for all } t \geqq 0 \text {. }
$$


Clearly $x(t-s)$ is a solution of the system (5) on $[s, s+T]$, and by the total stability of $u(t)$ we should have

$$
\left\|u_{t}-x_{t-s}\right\|<\varepsilon \text { on }[s, s+T]
$$

or

$$
\left\|u_{t+s}-x_{t}\right\|<\varepsilon \text { on }[0, T] \text {. }
$$

Thus there arises a contradiction, which shows that $u(t)$ is stable under disturbances from $H(F)$ with respect to $K$.

THEOREM 3. Let $u(t)$ be stable under disturbances from $H(F)$ with respect to $K$, and let the compact set $K \subset \bar{C}_{B} *$ be the one given in the proof of Lemma 2. Then the solutions in $H(u)$ are uniformly stable with a common number $\delta(\cdot)$.

ProOF. Let $(\gamma(\cdot), \eta(\cdot))$ be the pair given in the above definition. First of all, we shall prove that for any $\varepsilon>0$, any $t_{0} \geqq 0$, any $(\tau, G) \in H(u, F)$ and any solution $x(t)$ of (4),

$$
\left\|x_{t_{0}}-v_{t_{0}}\right\|<\frac{1}{2} \gamma\left(\frac{\varepsilon}{2}\right), x_{t_{0}} \in K \text { imply }\left\|x_{t}-v_{t}\right\|<\varepsilon \text { for all } t \geqq t_{0} .
$$

If not, then there exist $(v, G) \in H(u, F), \varepsilon>0, t_{0} \geqq 0$, a solution $x(t)$ of (4) and $\boldsymbol{\tau}>t_{0}$ satisfying

$$
\left\|x_{t_{0}}-v_{t_{0}}\right\|<\frac{1}{2} \gamma\left(\frac{\varepsilon}{2}\right), x_{t_{0}} \in K \text { and }\left\|x_{\tau}-v_{\tau}\right\|=\varepsilon .
$$

By replacing. $\left(\tau\left(t+t_{0}\right), G\left(t+t_{0}, \phi\right)\right)$ by $(v(t), G(t, \phi))$, we can assume $t_{0}=0$. Since $(v, G) \in H(u, F)$, there exists an $s \geqq 0$ such that

$$
\rho^{*}\left(F^{s}, G ; t\right)<\eta\left(\frac{\varepsilon}{2}\right) \text { zand }\left\|u_{t+s}-v_{t}\right\|<\frac{1}{2} \gamma\left(\frac{\varepsilon}{2}\right) \text { for } t \in[0, \boldsymbol{\tau}] .
$$

Since $x_{0} \in K$ and

$$
\left\|x_{0}-u_{s}\right\| \leqq\left\|x_{0}-v_{0}\right\|+\left\|v_{0}-u_{s}\right\|<\gamma\left(\frac{\varepsilon}{2}\right),
$$

we should have

$$
\left\|x_{t}-u_{t+s}\right\|<\frac{\varepsilon}{2} \text { for all } t \in[0, \tau]
$$


by the stability under disturbances from $H(F)$ of $u(t)$. Hence

$$
\left\|x_{t}-v_{t}\right\| \leqq\left\|x_{\imath}-u_{t+s}\right\|+\left\|u_{t+s}-v_{\imath}\right\|<\varepsilon \text { for all } t \in[0, \tau],
$$

from which there arises a contradiction at $t=\tau$. This proves the assertion (10).

Furthermore, from (10) we can easily prove that the solutions in $H(u)$. are unique for initial conditions, because $v_{t} \in K$ for all $v(t) \in H(u)$ and for all $t \geqq 0$. Therefore, from the fact that $H(v) \subset H(u)$ for every $v \in H(u)$, it follows that the solutions in $H(v)$ are unique for initial conditions. Hence, by Lemma 1 we can prove that

$$
\left\|x_{t_{0}+h}-v_{t_{0}+h}\right\|<\frac{1}{2} \gamma\left(\frac{\varepsilon}{2}\right) \text { and } x_{t_{0}+h} \in K
$$

whenever

$$
\left\|x_{t_{0}}-v_{t_{0}}\right\|<\delta(\varepsilon)=\delta_{1}\left(-\frac{1}{2} \gamma\left(\frac{\varepsilon}{2}\right), h\right)
$$

where $\delta_{1}$ is the one given in Lemma 1. By modifying the proof of Lemma 6 in [2], it is not difficult to see that $\delta_{1}$ can be chosen independently of each $v$ in $H(u)$, since $H(u)$ is compact. This completes the proof.

The following corollary is an immediate consequence of Theorems 2 and 3, where we consider the compact set $K$ given in the proof of Lemma 2 .

COROLLARY. If $u(t)$ is totally stable, then the solutions in $H(u)$ are uniformly stable with a common number $\delta(\cdot)$.

REMARK. As is clear in the proofs of Theorems 2 and 3, the relation (7) in the definition can be replaced by the simpler relation

$$
|G(t, \phi)-F(t+s, \phi)|<\eta(\varepsilon) \text { for all }(t, \phi) \in[0, T] \times K \text {. }
$$

However, in this case, the definition would be slightly different from the one given in [2] or [4], even for an almost periodic system.

4. Example. Let $f(x)$ be defined by

$$
f(x)= \begin{cases}2 \sqrt{|n x-1|} & \frac{2}{2 n+1} \leqq x \leqq \frac{2}{2 n-1}(n=1,2, \cdots), \\ 0 & x=0, \\ -f(-x) & x<0 .\end{cases}
$$

Then easily we can see that the zero solution of the system 


$$
\dot{x}=f(x)
$$

is not unique for initial conditions. Let $a(t)=\sum_{k=0}^{\omega} a_{k}(t)$ be the almost periodic function (see [4]), where $a_{0}(t) \equiv 1$ and $a_{k}(t)$ is a periodic function of period $2^{k}$ such that

$$
a_{k}(t)=\left\{\begin{array}{ll}
0 & 0<t<2^{k-1}, \\
-\frac{1}{2^{k}} & 2^{k-1} \leqq t \leqq 2^{k}
\end{array} \quad(k=1,2, \cdots)\right.
$$

(the continuity can be given by a slight modification), and consider the system. of a ordinary differential equation

$$
\dot{x}=F(t, x)
$$

where

$$
F(t, x)= \begin{cases}f(x)-c a(t) \sqrt{ } x & x \geqq 0, \\ -F(t,-x) & x<0\end{cases}
$$

for a constant $c>2 \sqrt{ } 2$.

Since $a(t)>0$ for all $t, F(t, 1 / n)<0$ for all $t$ and $n=1,2, \cdots$, which implies that any solution of (11) can not cross the line $x=1 / n$ upwards for $n=1,2, \cdots$. Hence the zero solution of (11) is uniformly stable. Furthermore, from the fact that $a(t) \geqq 1 / 2$ for $2 m<t<2 m+1$ and $f(x) \leqq \sqrt{2 x}$, it follows that

$$
F(t, x) \leqq\left(\sqrt{ } 2-\frac{c}{2}\right) \sqrt{ } x<0 \text { for } x>0 \text { and } 2 m<t<2 m+1
$$

and hence we can see that every solution of (11) starting from a neighborhood of $x=0$ tends to zero as $t \rightarrow \infty$ and, more precisely, that the zero solution of (11) is uniformly asymptotically stable. On the other hand, since

$$
a\left(t+2^{m}-1\right) \rightarrow 0 \text { as } m \rightarrow \infty \text { for } 0 \leqq t \leqq 1 \text {, }
$$

there exists a $G(t, x) \in H(F)$ such that $G(t, x)=f(x)$ for all $t \in[0,1]$. Therefore 
the zero solution of the system

$$
\dot{x}=G(t, x)
$$

is not unique for initial conditions, and consequently it is not uniformly stable. Hence the zero solution of (11) is neither totally stable nor stable under disturbances from $H(F)$.

\section{REFERENCES}

[1] J. KATO AND T. YoshizAWA, A relationship between uniformly asymptotic stability and total stability, Funkcial. Ekvac., 12(1969), 233-238.

[2] T. YoshizAwA, Asymptotically almost periodic solutions of an almost periodic system, Funkcial. Ekvac., 12(1969), 23-40.

[3] A.STRAUSS AND J. A. YORKE, Perturbing uniform asymptotically stable nonlinear systems, J. Differential Equations, 6(1969), 452-483.

[4] G. R. Sell, Nonautonomous differential equations and topological dynamics, I, II, Trans. Amer. Math. Soc., 127(1967), 241-262; 263-283.

MATHEMATICAL INSTITUTE

TÒHOKU UNIVERSITY

SENDAI, JAPAN 